\title{
IgA Nephropathy: Actually an Inherited Disease?
}

\section{Dimitrios Kirmizis*}

Department of Nephrology, Hippokration General Hospital, Aristotle University, Thessaloniki, Greece

\begin{abstract}
IgA Nephropathy (IgAN) is the most common primary glomerulonephritis worldwide. Over the last years strong evidence suggest the existence of genetic factors in the development and/or progression of IgAN. IgAN does not exhibit classic single-gene Mendelian but rather a complex genetic inheritance pattern. Until now, two basic approaches have been used in genetic studies of IgAN: Genome-Wide linkage Analysis Studies (GWAS) and candidate-gene association studies. Many candidates have been proposed, but most were studied in the context of IgAN progression rather than causality. In addition, in part due to our lack of knowledge about disease pathogenesis, most candidates were predicated on sparse a priori evidence for involvement in IgAN. Overall, candidate-gene studies for IgAN have been largely unrevealing. The advantage of GWAS, on the other hand, is that they do not require a priori assumptions about disease pathogenesis. To date six GWAS of familial IgAN have identified several loci located on chromosomes $2,4,6,7,12$ and 17 . The functional mapping of genes involved in the disease is anticipated to proceed further from the identification of susceptibility loci identified by linkage analysis to the isolation of candidate genes within gene diseasesusceptibility loci. In conclusion, inheritance of IgAN follows a complex genetic pattern, most probably autosomal dominant inheritance with incomplete penetrance. It seems that several genetic loci contribute significantly to the disease susceptibility that underlies the primary immunologic defects observed in IgAN.
\end{abstract}

Keywords: IgA nephropathy; Genetic studies; Inherited disease; Susceptibility; Familial disease

\section{Introduction}

IgAN has been considered for long a benign disease. Today it is clear that as many as $30-40 \%$ of the patients develop renal disease, with ensuing significant social and financial impact. In western societies, IgAN is responsible of as much as $7-10 \%$ of End-Stage Renal Disease (ESRD) patients on substitution treatment. IgAN occurs at any age, but most commonly the age of onset is in the second or third decade of life. Males are more often affected than females, with a male:female ratio of 2:1. Most patients with IgAN present microscopic hematuria with or without mild proteinuria. About $40 \%$ of patients have episodes of macroscopic hematuria. This is sometimes preceded by infections, most commonly upper respiratory tract infections, a phenomenon known as "synpharyngitic" hematuria. Other infections like gastrointestinal or urinary tract infections have also been reported to precede macroscopic hematuria. Proteinuria is common and can vary from mild proteinuria to nephrotic syndrome. Recurrence of IgAN after renal transplantation is common. This finding, along with the observation that IgA depositions disappear from a kidney of an IgAN patient, after transplantation of this kidney to a non IgAN patient, are suggestive of a rather systemic disease.

\section{Genetic Basis of IgAN}

The strongest evidence for the existence of genetic factors in the development and/or progression of IgAN comes from descriptions of familial IgAN [1] whereas, interestingly, recent studies suggest that familial and sporadic IgAN may share a common pathogenic mechanism and similar outcomes [2]. The genetic susceptibility may be independent of environmental factors and may reflect an inherited susceptibility to develop mesangial glomerulonephritis. IgAN does not exhibit classic single-gene Mendelian inheritance pattern [3]. The complex genetic pattern of IgAN is reflected by the multiple pathways involved in its immunopathogenesis, namely multiple discrete immunologic abnormalities related to the abnormal overproduction and release of mucosal polymeric IgA1 (pIgA1) in the systemic compartment and possibly other protein functional abnormalities related to a propensity for mesangial deposition of pIgA1. It is therefore probable that the disease is actually caused by specific SingleNucleotide Polymorphism (SNP) alleles in non-coding regions or synonymous SNPs in coding regions at identified IGAN loci rather than by "classic" nonsense/missense/splice site mutations and deletions/ insertions. Therefore, the actual problem is probably the alterations by SNPs of the transcriptional activity of a disease gene and/or messenger RNA stability and, therefore, of the expression level of the encoded protein. It is interesting that recent studies indicate that 30\% to $50 \%$ of human genes with coding SNPs can present allelic variation in gene expression $[4,5]$. To this context, the most comprehensive assumption is that several genetic loci convey the primary immunologic defects observed in IgAN and, therefore, contribute significantly to the disease susceptibility. Each locus may occur at a different prevalence rate in different racial/ethnic groups. Variations at these major genetic loci may not be sufficient for the development and progression of IgAN and the contribution from a potentially large number of modifying genes with modest genetic effects but high prevalence is probably needed as well. The various allelic combinations of these loci may underlie the different disease phenotypes (disease development and progression, nephritic $v s$. nephrotic clinical presentation, histopathologic subclass, severity of disease, responsiveness of proteinuria to AngiotensinConverting Enzyme (ACE) inhibitors and/or Angiotensin II Receptor Blockers (ARBs), etc.) observed in IgAN. For diseases with complex genetic pattern, it has been shown that the optimal analysis approach is the combination of linkage, association and sequence approaches. Until now, two basic approaches have been used in genetic studies of IgAN: a) genome-wide linkage analysis study, a methodology that has

*Corresponding author: Dr. Dimitrios Kirmizis, Department of Nephrology, Hippokration General Hospital, Aristotle University, Thessaloniki, Greece, Tel: +30 2310-938977; Fax: +30-2310-938979; E-mail: dkirmizis@yahoo.co.uk

Received November 19, 2012; Accepted December 28, 2012; Published December 31, 2012

Citation: Kirmizis D (2013) IgA Nephropathy: Actually an Inherited Disease? J Nephrol Therapeut S11: 001. doi:10.4172/2161-0959.S11-001

Copyright: @ 2013 Kirmizis D. This is an open-access article distributed under the terms of the Creative Commons Attribution License, which permits unrestricted use, distribution, and reproduction in any medium, provided the original author and source are credited. 
been used successfully to identify major disease/susceptibility loci, b) candidate-gene association study, mainly used to identify altered genes with modest genetic effects but high prevalence.

However, it is important to realize that genetic studies of IgAN are faced with significant challenges. Thus, familial forms of IgAN are frequently underrecognized because the associated urinary abnormalities in affected family members are often mild or intermittent. Moreover, once familial disease is documented, systematic screening by renal biopsy cannot be justified among asymptomatic at-risk relatives, necessitating reliance on less accurate phenotypes, such as microscopic hematuria, to diagnose affection. Additionally, IgAN has been observed to co-occur in families with Thin Basement Membrane Disease (TBMD), an autosomal dominant disease caused by heterozygous mutations in the collagen type IV genes (COL4A3/ COL4A4) [6]. Short of kidney biopsy or direct sequencing of the very large collagen genes, TBMD cannot be reliably excluded among relatives of IgAN patients. Finally, because urinary abnormalities may manifest intermittently, one also cannot unequivocally classify at-risk relatives as unaffected, necessitating affected-only linkage analysis. The inability to classify affected and unaffected individuals accurately is commonly encountered in linkage studies of complex traits, leading to decreased study power. Increasing sample size by including additional families is also not necessarily helpful in these situations because the diagnosis of IgAN likely encompasses several disease subsets, such that expansion to larger sample size can paradoxically reduce analytic power due to increased heterogeneity $[7,8]$.

\section{Genome-wide linkage analysis studies}

Genome-wide linkage analyses involve recruitment of families with multiple affected individuals and are used successfully to identify major disease/susceptibility genes but have limited power to detect genes of modest effect. The advantage of genome-wide linkage studies is that they do not require a priori assumption about disease pathogenesis. These studies are very sensitive to phenotype misspecification; however their power is limited to detecting rare genetic variants with a relatively large effect on the risk of disease. To date, six genome-wide linkage studies of familial IgAN have been reported [9-15]. Families in these studies have all been ascertained via at least two cases with biopsy-documented IgAN, with additional family members diagnosed as affected based on clinical evidence (renal failure or multiple documentation of hematuria/ proteinuria). In the first study, 30 families with two or more affected members were examined [9]; multipoint linkage analysis under the assumption of genetic heterogeneity yielded a peak LOD score of 5.6 on chromosome 6q22-23 (locus named IGAN1), with $60 \%$ of families linked. The remainder of families linked to chromosome 3p24-23 with a suggestive LOD of 2.8 . This study demonstrated that IgAN is genetically heterogeneous but argued for the existence of a single locus with a major effect in some families. The second GWAS was part of the European IgAN Consortium coordinated by Schena et al. $[10,11]$ and comprised of at minimum 72 multiplex extended pedigrees, 159 trios, and 1,068 cases and 1,040 matched controls. All subjects enrolled were white and belonged to various geographic areas from Germany, Italy, and Greece. The European IgAN Consortium performed the first GWAS involving 22 Italian multiplex IgAN families [10]. A total of 186 individuals (59 affected and 127 unaffected) were genotyped and included in a twostage linkage analysis. The regions 4q26-31 and 17q12-22 exhibited the strongest evidence of linkage by non-parametric analysis (best $\mathrm{p}$ values of 0.0025 and 0.0045 , respectively). These localizations were also supported by multipoint parametric analysis where a peak LOD score of $1.83(\alpha=0.50)$ and of $2.56(\alpha=0.65)$, respectively, were obtained using the affected only dominant model, and by allowing for the presence of genetic heterogeneity. These regions became the second (IGAN2) and third (IGAN3) genetic locus candidates to contain causative and/or susceptibility genes for familial IgAN. The above results provide further evidence for genetic heterogeneity among IgAN families. Evidence of linkage to multiple chromosomal regions is consistent with both an oligo/polygenic and a multiple susceptibility gene model for familial IgAN with small/moderate effects in determining the pathological phenotype. The analysis of the known genes located in these two novel loci identified some potential candidate genes such as the transient receptor potential channel 3 (TRPC3) genes, the interleukin-2 (IL-2) gene, and the IL-21 gene located in 4q26-31, which could be largely involved in the unbalanced Th1/Th2 immune response reported in IgAN patients.

The third linkage scan was based on a unique large pedigree with 14 affected relatives (two individuals with biopsy defined diagnosis and 12 with hematuria/proteinuria on urine dipstick) [12]. Linkage to chromosome $2 \mathrm{q} 36$ was detected with a maximal multipoint LOD score of 3.47. Most reported linkage intervals did not contain obvious candidate genes, but the $2 \mathrm{q} 36$ locus encompasses the COL4A3 and COL4A4 genes, which are mutated in TBMD. Together with the high penetrance of hematuria, this finding suggests that affected individuals in the 2q36-linked family may belong to an IgAN subtype that overlaps with TBMD. The fourth genome-wide analysis, carried out in a cohort of IgAN patients selected from the UK Glomerulonephritis DNA Bank, the region of the MHC (Major Histocompatibility Complex) [13]. The strongest association signal included a combination of DQ loci and HLA-B. This study suggests that HLA region contains some alleles that predispose to the disease in the UK population. In conclusion, four genome-wide linkage studies, carried out in four different IgAN patient populations, demonstrate different chromosomal traits linked to the disease. Recently, in a GWAS conducted by Gharavi et al. [14] in Chinese and European IgAN patients five loci were traced (3 loci in the Major Histocompatibility Complex (MHC) region on chromosome 6p21, a common deletion CGHR1 and CFHR3 on chromosome 1q32 and a locus on chromosome 22q12 to be related with IgAN and account for the $4-7 \%$ of the genetic heterogeneity of the disease. Finally, the most recent GWAS was conducted by Yu et al. [15], who performed a two-stage genome-wide association study of IgA nephropathy (IgAN) in Han Chinese, with 1,434 affected individuals and 4,270 controls in the discovery phase and follow-up of the top 61 SNPs in an additional 2,703 cases and 3,464 controls. They identified associations at $17 \mathrm{p} 13$ and $8 \mathrm{p} 23$, that implicated the genes encoding tumor necrosis factor (TNFSF13) and a-defensin (DEFA) as susceptibility genes. In addition, they found multiple associations in the MHC region and confirmed a previously reported association at 22q12. They also found that rs660895 was associated with clinical subtypes of IgAN, proteinuria and IgA levels. These findings are consistent with the hypothesis that IgAN is associated with variants near genes involved in innate immunity and inflammation.

\section{Candidate-gene association studies}

Candidate-gene association studies examine polymorphisms in only specific genes that are selected based on a priori assumption about their involvement in the disease pathogenesis, and they are highly sensitive to population stratification, multiple testing, and 
reporting bias. As a result, most candidate-gene association studies in the literature have not been replicated [3,16-18], an issue which questions the validity and the methodology used in these studies [19]. Not surprisingly, candidate-gene studies for IgAN have also been largely unrevealing. Many candidate genes have been proposed, but for most of them no solid a priori evidence for their involvement in IgAN existed, whereas most were studied in the context of IgAN progression rather than causality. Over the last 15 years, amongst over 120 candidate-gene association studies for IgAN published in the English literature [20], one third examined genetic polymorphisms in association with susceptibility to IgAN, another third examined an association with disease severity, progression, or complications, and the rest one third examined both susceptibility and risk of progression. Approximately one third of all studies involved polymorphisms in the Rennin-Angiotensin-Aldosterone System (RAAS), with the majority focusing on the role the Angiotensin-I Converting Enzyme (ACE) gene Insertion/Deletion (I/D) polymorphism in the development and/or progression of IgAN, as well as many other common human diseases, including cardiovascular disease, complications of diabetes such as retinopathy and nephropathy, glomerular, tubulointerstitial, and renal cystic renal diseases, and even renal allograft survival [17,18,21]. There have been numerous population-based studies that either support or refute an association between the $\mathrm{D}$ allele and progression of renal disease in these conditions $[17,18]$. Recent meta-analyses have concluded that the $\mathrm{D}$ allele is not associated with renal disease progression in patients with IgAN or diabetic nephropathy [21,22]. Despite more than a dozen generally small genetic case-control studies of the ACE I/D polymorphism in both white and Asian IgAN cohorts have been done, no definite conclusions can be drawn from them regarding the association between the $\mathrm{D}$ allele or $\mathrm{DD}$ genotype and development and/or progression of IgAN. Population-based genetic association studies of other genes encoding proteins in the RAAS, such as angiotensinogen, the angiotensin II type 1 receptor, renin and aldosterone synthase, etc., have also yielded conflicting results [23].

In general, most of these studies were of poor quality and severely underpowered and, therefore, negative findings were almost universally inconclusive. Thus far, only one group attempted to survey the entire genome, yet the results have not been replicated, are inconclusive and difficult to interpret, as long as an underpowered cohort was studied with inadequate coverage of $\sim 80,000$ SNPs $[24,25]$. Moreover, $77 \%$ of all published candidate-gene studies reported positive findings, an observation that is likely explained by a combination of high rate of false positives and a strong publication bias. Most findings were not reproduced in other populations. Overall, to date, very few studies examining candidate genes have employed the familybased Transmission Disequilibrium Test (TDT) methodology, or the analysis of "at-risk" haplotypes or the Family-Based Association Test (FBAT), reflecting the emergence of the first studies to attempt to satisfy minimum criteria for a valid association study. A family- and haplotype-based association study employing the TDT methodology has shown that 2093C and 2180T SNP variants in the 3'-untranslated region of the Megsin gene were significantly more frequently transmitted from heterozygous parents to patients than expected in the extended TDT analysis (increased co-transmission in 232 Chinese families). In addition, Haplotype Relative Risk (HRR) analyses showed that these same SNP alleles were more often transmitted to patients [26]. The same group using a similar approach recently reported that the Megsin 23167G SNP variant is associated with both susceptibility and progression of IgAN in 435 Chinese patients and their family members using TDT and HRR analyses [27]. The GG genotype was found to be associated with severe histologic lesions and disease progression. Megsin is a member of the serpin (serine proteinase inhibitor) superfamily that is upregulated in the context of mesangial proliferation and extracellular matrix expansion in IgAN, and therefore represents a strong candidate gene for susceptibility to IgAN. Lately, the gene encoding Cosmc, a C1Gal-T1 chaperone protein which also mediates IgA O-galactosylation was studied as a candidate gene involved in the pathogenesis of IgAN but no evidence for a role for Cosmc mutations was reported [28].

A family-based association study performed by IgAN Consortium, including 53 patients, 45 complete trios, 4 incomplete trios and 36 discordant siblings, evaluated the impact of some Th1/Th2/Th3/ TR-type lymphocyte and monocyte/macrophage cytokines on IgAN susceptibility [29]. Cytokine gene polymorphisms with a potential regulatory role on their production were investigated using the FBAT: IFN $\gamma$ intron-1 CA-repeat at position 1349-1373; IL-13 - 1055C/T; TGF $\beta$ 915G/C; IL-10 5'-proximal and distal microsatellites; TNFa-308G/A, $-238 \mathrm{G} / \mathrm{A}$. The FBAT multi-allelic analysis showed an association between IFN $\gamma$ polymorphism and susceptibility to IgAN, whereas the bi-allelic analysis showed that the 13-CA repeat allele was preferentially transmitted to the affected individuals. The in vitro production of IFN $\gamma$ evaluated in PBMC from 10 genotyped patients demonstrated a correlation between the $+874 \mathrm{~A}$ allele and a lower production of IFN $\gamma$.

Furthermore, consistent with the known role of NF- $\mathrm{KB}$ in the transcriptional regulation of the INF $\gamma$ gene, the $+874 \mathrm{~A}$ variant was associated with transcriptional downregulation of INF $\gamma$ gene promoter activity. The occurrence of the $+874 \mathrm{~A}$ variant is responsible for the low production of IFN $\gamma$ and predisposes to a preferential Th2-mediated immune response. The predominance of this variant in individuals with IgAN may be responsible for the onset of the disease. This unbalanced $\mathrm{Th} 2$ cytokine production in response to upper respiratory tract infections may be a significant pathogenic factor in human IgAN The prevalence of Th2 cytokines may also explain the abnormality in IgA1 glycosylation occurring in IgAN patients and the concomitant formation of circulating IgA 1-IgG immune complexes. Hyperfunction of Th2 cells and cytokine polarity are linked to a more nephritogenic pattern of IgA1 glycosylation in the animal model, and the decreased glycosylation of IgA1 elicited by Th2 cytokines is blunted in vitro by the addition of IFN $\gamma$ [30]. The core $1 \beta 1,3$-galactosyltransferase (C1Gal-T1) is suspected to be involved in the abnormal glycosylation process of IgA1 in IgAN. With the genetic characterization of the enzymes responsible for O-glycosylation of $\mathrm{IgA} 1$, it has been possible to study changes in the $\mathrm{O}$-glycosylation of IgA1 at a genetic level. Most recently two groups $[31,32]$ have independently found that SNPs in the C1Gal-T1 gene (genotype 1365G/G) confer genetic susceptibility to IgAN, yet not to the progression of the disease as well, in Chinese and Italian populations, albeit it is not clear how these polymorphisms relate to changes in the functional activity of $\mathrm{C} 1 \mathrm{Gal}-\mathrm{T} 1$.

\section{Clinical Implications}

The above results provide further evidence for genetic heterogeneity among IgAN families. Evidence of linkage to multiple chromosomal regions is consistent with both an oligo/polygenic and a multiple susceptibility gene model for familial IgAN with small/moderate effects in determining the pathological phenotype. Susceptibility to IgA nephropathy has a genetic component to varying degrees, with significant diversity between different geographical areas. Familial forms of IgAN may derive from a mutation of a specific gene occurring 
in a founder several generations ago. Although several genetic loci are strongly associated with IgAN, the implication of the "guilty" genes is yet far from being well documented. It seems probable that the disease is inherited as an autosomal-dominant trait with highly variable penetrance.

But what about the vast majority of IgAN cases which seem to be sporadic? Although many of these cases may actually have a less apparent genetic basis, the existing evidence is rather deficient on this issue. To what extent genetic and environmental factors contribute to the pathogenesis of IgAN is still not known with accuracy. At present, genetic testing does not appear to have much diagnostic value except in clear-cut familial cases, because many loci are involved, and renal biopsy remains the golden diagnostic standard. It is noteworthy that most genetic studies actually examined susceptibility to the clinical expression of disease rather than susceptibility to the mesangial IgA deposition itself. On the basis of these preliminary findings, however, the extended genetic studies under way are anticipated to shed light to the pathogenetic mechanisms and allow for the earlier diagnosis of the disease or even the genetic susceptibility for the disease, more efficient preventive policies and more targeted and fruitful treatment in the near future.

\section{Conclusions}

Despite the fact that clear-cut genetic susceptibility can be found in the minority of IgAN cases, this might be attributed at least in part to its complex genetic pattern, most probably autosomal dominant pattern with incomplete penetrance. Incomplete penetrance might be attributed to the need for the presence of additional genetic and/ or environmental factors for the clinical manifestation of the disease. To date several loci have been associated with IgAN by different groups, although there is still long road ahead to document definitely the implication of the respective candidate genes. These preliminary findings, however, have created great expectations that the extended genetic studies under way with the use of high technology high throughput assays will shed light to pathogenetic mechanisms of the disease and allow the earlier diagnosis of the disease or even the genetic susceptibility for the disease, more efficient preventive policies and more targeted and fruitful treatment in the near future.

\section{References}

1. Julian BA, Quiggins PA, Thompson JS, Woodford SY, Gleason K, et al. (1985) Familial IgA nephropathy. Evidence of an inherited mechanism of disease. $\mathrm{N}$ Engl J Med 312: 202-208

2. Izzi C, Ravani P, Torres D, Prati E, Viola BF, et al. (2006) IgA nephropathy: the presence of familial disease does not confer an increased risk for progression. Am J Kidney Dis 47: 761-769.

3. Frimat L, Kessler M (2002) Controversies concerning the importance of genetic polymorphism in IgA nephropathy. Nephrol Dial Transplant 17: 542-545.

4. Hoogendoorn B, Coleman SL, Guy CA, Smith K, Bowen T, et al. (2003) Functional analysis of human promoter polymorphisms. Hum Mol Genet 12: 2249-2254.

5. Lo HS, Wang Z, Hu Y, Yang HH, Gere S, et al. (2003) Allelic variation in gene expression is common in the human genome. Genome Res 13: 1855-1862.

6. Frascá GM, Soverini L, Gharavi AG, Lifton RP, Canova C, et al. (2004) Thin basement membrane disease in patients with familial IgA nephropathy. J Nephrol 17: 778-785.

7. Durner M, Greenberg DA, Hodge SE (1992) Inter- and intrafamilia heterogeneity: effective sampling strategies and comparison of analysis methods. Am J Hum Genet 51: 859-870.

8. Cavalli-Sforza LL, King MC (1986) Detecting linkage for genetically heterogeneous diseases and detecting heterogeneity with linkage data. Am J Hum Genet 38: 599-616.

9. Gharavi AG, Yan Y, Scolari F, Schena FP, Frasca GM, et al. (2000) IgA nephropathy, the most common cause of glomerulonephritis, is linked to $6 \mathrm{q} 22$ 23. Nat Genet 26: 354-357.

10. Bisceglia L, Cerullo G, Forabosco P, Torres DD, Scolari F, et al. (2006) Genetic heterogeneity in Italian families with IgA nephropathy: suggestive linkage for two novel IgA nephropathy loci. Am J Hum Genet 79: 1130-1134.

11. Schena FP, Cerullo G, Torres DD, Scolari F, Foramitti M, et al. (2005) The IgA nephropathy Biobank. An important starting point for the genetic dissection of a complex trait. BMC Nephrol 6: 14

12. Paterson AD, Liu XQ, Wang K, Magistroni R, Song X, et al. (2007) Genome wide linkage scan of a large family with IgA nephropathy localizes a nove susceptibility locus to chromosome 2q36. J Am Soc Nephrol 18: 2408-2415.

13. Feehally J, Farrall M, Boland A, Gale DP, Gut I, et al. (2010) HLA has strongest association with IgA nephropathy in genome-wide analysis. J Am Soc Nephrol 21: $1791-1797$

14. Gharavi AG, Kiryluk K, Choi M, Li Y, Hou P, et al. (2011) Genome-wide association study identifies susceptibility loci for IgA nephropathy. Nat Genet 43: $321-327$

15. Yu XQ, Li M, Zhang H, Low HQ, Wei X, et al. (2011) A genome-wide association study in Han Chinese identifies multiple susceptibility loci for IgA nephropathy. Nat Genet 44: 178-182.

16. Ioannidis JP, Ntzani EE, Trikalinos TA, Contopoulos-loannidis DG (2001) Replication validity of genetic association studies. Nat Genet 29: 306-309.

17. Hsu SI, Ramirez SB, Winn MP, Bonventre JV, Owen WF (2000) Evidence for genetic factors in the development and progression of IgA nephropathy. Kidney Int 57: 1818-1835.

18. Hsu SI (2001) The molecular pathogenesis and experimental therapy of $\lg A$ nephropathy: recent advances and future directions. Curr Mol Med 1: 183-196.

19. Hsu S, Feehally J (2007) The Molecular Basis of IgA Nephropathy. In: Mount DB, Pollak MR (eds) Molecular and Genetic Basis of Renal Disease: A Companion to Brenner \& Rector's The Kidney, Elsevier Health Sciences Philadelphia.

20. Kiryluk K, Julian BA, Wyatt RJ, Scolari F, Zhang H, et al. (2010) Genetic studie of IgA nephropathy: past, present, and future. Pediatr Nephrol 25: 2257-2268.

21. Schena FP, D’Altri C, Cerullo G, Manno C, Gesualdo L (2001) ACE gene polymorphism and IgA nephropathy: an ethnically homogeneous study and a meta-analysis. Kidney Int 60: 732-740.

22. Kunz R, Bork JP, Fritsche L, Ringel J, Sharma AM (1998) Association between the angiotensin-converting enzyme-insertion/deletion polymorphism and diabetic nephropathy: a methodologic appraisal and systematic review. J Am Soc Nephrol 9: 1653-1663.

23. Poch E, González D, Giner V, Bragulat E, Coca A, et al. (2001) Molecular basis of salt sensitivity in human hypertension. Evaluation of renin-angiotensinaldosterone system gene polymorphisms. Hypertension 38: 1204-1209.

24. Obara W, lida A, Suzuki Y, Tanaka T, Akiyama F, et al. (2003) Association of single-nucleotide polymorphisms in the polymeric immunoglobulin receptor gene with immunoglobulin A nephropathy (IgAN) in Japanese patients. J Hum Genet 48: 293-299.

25. Ohtsubo S, lida A, Nitta K, Tanaka T, Yamada R, et al (2005) Association of a single-nucleotide polymorphism in the immunoglobulin mu-binding protein 2 gene with immunoglobulin A nephropathy. J Hum Genet 50: 30-35.

26. Li YJ, Du Y, Li CX, Guo H, Leung JC, et al. (2004) Family-based association study showing that immunoglobulin A nephropathy is associated with the polymorphisms 2093C and 2180T in the 3' untranslated region of the Megsin gene. J Am Soc Nephrol 15: 1739-1743.

27. Takei T, Hiraoka M, Nitta K, Uchida K, Deushi M, et al. (2006) Functiona impact of IgA nephropathy-associated selectin gene haplotype on leukocyteendothelial interaction. Immunogenetics 58: 355-361.

28. Malycha F, Eggermann T, Hristov M, Schena FP, Mertens PR, et al. (2009) No evidence for a role of cosmc-chaperone mutations in European IgA nephropathy patients. Nephrol Dial Transplant 24: 321-324.

29. Schena FP, Cerullo G, Torres DD, Scolari F, Foramitti M, et al. (2006) Role of 
interferon-gamma gene polymorphisms in susceptibility to IgA nephropathy: a family based association study. Eur J Hum Genet 14: 488-496.

30. Ebihara I, Hirayama K, Yamamoto S, Muro K, Yamagata K, et al. (2001) Th2 predominance at the single-cell level in patients with IgA nephropathy. Nephrol Dial Transplant 16: 1783-1789.

31. Pirulli D, Crovella S, Ulivi S, Zadro C, Bertok S, et al. (2009) Genetic variant of C1GalT1 contributes to the susceptibility to IgA nephropathy. J Nephrol 22 : 152-159.

32. Li GS, Zhang H, Lv JC, Shen Y, Wang HY (2007) Variants of C1GALT1 gene are associated with the genetic susceptibility to IgA nephropathy. Kidney Int 71: $448-453$ 